\title{
MECHANICAL LIMITATIONS IN HIGH AVERAGE POWER LINACS
}

A. Walker and W. J. Gallagher i

Boeing Aerospace Co., Seattle, WA 98124

The intention of providing high average power beams being presently proposed involves the deposition of large amounts of heat in the acceleration waveguide; the thermal gradients which result can permanently detune the RF structure. This paper presents an outline of the method of analyzing such a problem, taking the disc-loaded transmission line as example.

The ficlds of the TM-OT acceleration mode in a disc-loaded, slow-wave structure are: 1

$$
\begin{aligned}
& E_{Z}=E_{0} j_{0}\left(k_{1} r\right) \sin \left(w t-k_{g} z\right) \\
& E_{r}=E_{0} \frac{k_{g}}{k_{1}} J_{1}\left(k_{1} r\right) \cos \left(w t-k_{g} z\right) \quad r<a \\
& \mathrm{~B}_{\phi}=\frac{\varepsilon_{0}}{n} \frac{k}{\mathrm{k}_{1}} \mathrm{~J}_{1}\left(\mathrm{k}_{1} r\right) \cos \left(w t-k_{g} z\right) \\
& \left.E_{Z}=-E_{0} \frac{J_{0}(k r) N_{0}(k b)-N_{0}(k r) J_{0}(k b)}{J_{0}(k a) N_{0}(k b)-N_{0}(k a) J_{0}(k b)} \sin w t\right) \\
& \left.B_{\phi}=\frac{E_{0}}{n} \frac{J_{l}(k r) N_{0}(k b)-N_{1}(k r) J_{0}(k b)}{J_{0}(k a) N_{0}(k b)-N_{0}(k a) J_{0}(k b)} \cos w t\right\} \\
& k^{2}=k_{1}{ }^{2}+k_{g}{ }^{2}, k=2 \pi / \lambda, k_{1}=k \frac{\left[\left(v_{p} / c\right)^{2}-1\right]^{2}}{v_{p} / c}, k_{g}=2 \pi / \lambda g
\end{aligned}
$$

Such fields are, of course, associated with currents in the metallic surfaces of the waveguide, which in consequence of surface resistivity generate heat.

Quantitatively the surface currents are equal in magnitude to the tangential magnetic field; thus the heat generated is $i 2 R_{s} / 2$ where $R_{s}$ is the surface resistivity (in ohms per square) and the factor 2 arises from the time average of the field.

This heat must flow through the metal substrate to the coolant; conductive heat flow is described by Fourier's equation,

$$
K \nabla^{2} T+p=\rho g \frac{\partial T}{\partial t}
$$

where $p$ is a heat source per unit volume, $k$ is the thermal conductivity, $\rho$ the density and $g$ the specific heat of the metal. Even in the steady state we require, therefore, a solution to Poisson's equation.

In the general case, where the heat source is a distributed function, a solution may be obtained by means of an inverse differential operator (integral operator). In particular, if $L$ is any ordinary linear differential operator and $u$ and $f$ are unknown and known functions, respectively, the nonhomogeneous equation

$$
\text { Lu }=f(x)
$$

can be solved if an inverse operator can be found. 2 There is always such an operator, the Green's function $G$, composable from the set of solutions of the homogeneous equation, which may be used as a kernel for integration to solve the nonhomogeneous equation. That is, solutions of eq( 3 ) are

$$
u=\int G(x, \xi) f(\xi) d \xi
$$

The Green's function which is the solution of Laplace's equation in cylindrical coordinates where there are only radial variations is

$$
\begin{aligned}
& G_{1}=\ln \frac{b}{r}, r<r_{0} \\
& G_{2}=\ln \frac{b}{r_{0}}, r>r_{0}
\end{aligned}
$$

In the actual physical case of this discussion heat is generated on the surface and the problem is to find the temperature in $r$ and $z$ coordinates (since there are no $\phi$-variations) but for simplicity, since the discs are thin, a solution in $r$ only (as if the heat were generated throughout the metal volume) will be of sufficient accuracy for engineering purposes. There is, of course, no such complication at the cavity walls; all the heat generated on the inside surface flows to the coolant, establishing a temperature drop

$$
T_{b}-T_{c}=\frac{p \ln \frac{c}{b}}{2 \pi K}
$$

where $p$ is the heat power generated per unit length, and $T_{c}$ is surface temperature at the coolant radius, $C$.

Equation (2) may now be put in the form of the solution to determine the temperature within the discs, ${ }^{3}$

$$
\begin{aligned}
& T(r)=\left(\frac{E_{0}}{n}\right)^{2} \frac{R_{S} 2}{K t}\left[\int_{a}^{r} r_{0} G_{1} p\left(r_{0}\right) d r_{0}+\int_{r}^{b} r_{0} G_{2} p\left(r_{0}\right) d r_{0}\right] \\
& =\left(\frac{E_{0}}{r}\right)^{2} \frac{2 R_{s}}{k+J_{0}^{2}(k a)}\left[\ln \frac{b}{a} \int_{a}^{r} r_{0} J_{1}{ }^{2}\left(k r_{0}\right) d r_{0}\right. \\
& \left.+\int_{r}^{b} r_{0} \ln \frac{b}{r_{0}} J_{1}^{2}\left(k r_{0}\right) d r_{0}\right] \\
& T(r)=\left(\frac{E_{0}}{n}\right)^{2} \frac{2 R_{s}}{k t J_{0}^{2}(k a)}\left[\frac{b^{2}}{2}\left(j_{0}^{2}(k b)+J_{1}^{2}(k b)\right)\right. \\
& -\frac{b}{4 k} J_{0}(k b) J_{1}(k b)+\left(\frac{J_{0}(k b)}{2 k}\right)^{2} \\
& -\frac{a^{2}}{2}\left(J_{0}^{2}(k a)+J_{1}^{2}(k a)\right) \ln \frac{b}{a}-\frac{a}{k} J_{0}(k a) J_{1}(k a) \ln \frac{b}{a} \\
& -\frac{r}{2 k} J_{0}(k r) J_{1}(k r)\left(\ln \frac{b}{r}-\frac{1}{2}\right)-\frac{r^{2}}{2}\left(J_{0}^{2}(k r)+J_{1}^{2}(k r)\right) \\
& \left.-\left(\frac{\mathrm{J}(k r)}{2 k}\right)^{2}\right] \\
& T_{a}-T_{b}=\left(\frac{E_{0}}{n}\right)^{2} \frac{2 R_{s}}{k t}\left[\frac{b^{2}}{2}\left(\frac{J_{1}(k b)}{J_{0}(k a)}\right)^{2}-\frac{a}{2 k} \frac{J_{1}(k a)}{J_{0}(k a)}\left(n n \frac{b}{a}-\frac{1}{2}\right)\right. \\
& \left.-\frac{a^{2}}{2}\left(1+\frac{J_{1}^{2}(k a)}{J_{0}^{2}(k a)}\right)-\frac{1}{4 k^{2}}\right]
\end{aligned}
$$


In the solution, eq(7), it has been assumed for simplicity that $J_{0}(k b)=0$ where $2 b$ is the inside waveguide diameter, that equal heat deposition occurs on both faces of the disc and that the thermal conductivity is not a function of temperature. aperture,

Integrating the fields over the area of the

$$
P=\int_{0}^{a} \operatorname{ErH} 2 \pi r d r=\frac{\pi E_{0}^{2} k^{2} a^{4}}{16 \eta}
$$

by which the temperature at the aperture (the hottest region) may be expressed in terms of the power flux so that the coefficient in eq(7) becomes

$$
\left(\frac{E_{0}}{\pi_{1}}\right)^{2} \frac{2 R_{s}}{K t}=\frac{32 P R_{s}}{\pi k^{2} a^{4} k t}
$$

It is nearly always useful to have some alternate confirmation that the order of magnitude derived from a theoretical calculation is reasonable; in some cases, as in the present one, the analyst does not have a "feel" for the answer. As a first approximation, to estimate whether the temperature rise at the disc aperture is tolerable (or should be investigated further), note that the ratio of heat losses in a disc to that in a spacer (periodic cavity wa11s) is approximately

$$
\begin{aligned}
R= & \frac{2 \int_{a}^{b} r J_{1}^{2}(k r) d r}{b d J_{1}^{2}(k b)}=\frac{b}{d}-\frac{a^{2}}{b d} \frac{J_{0}^{2}(k a)+J_{1}^{2}(k a)}{J_{1}^{2}(k b)} \\
& -\frac{2 a}{b k d} \frac{J_{0}(k a) J_{1}(k a)}{J_{1}{ }^{2} k b}=\frac{n}{2.61}, d=p-t
\end{aligned}
$$

which generally is of the order of unity, with most of the heat generated in the disc occurring near the outer radius. If the power attenuation per unit length is $2 \mathrm{I}$ and the power flux $P$ (including the duty factor), the heat power transferred by each disc is R2IPA/ $n$, $n$ being the number of discs per wavelength. It may be assumed, as an extreme case, that all the heat power generated in a disc must flow through the disc from the aperture to its outer rim. The cylindrical heat power transfer through a plate of thickness $t$, thermal conductivity, $k$, having an inner and outer temperature $T_{i}$ and $T_{0}$ and radi $i$ a and $b$, respectively, is

$$
P=2 \pi k t\left(T_{i}-T_{0}\right) \ln \frac{b}{a}
$$

For example, the SLAC $3 \mathrm{~m}$ constant gradient waveguide $\left(2 \mathrm{I}_{\mathrm{O}} \mathrm{L}=.684, \mathrm{t}=.584 \mathrm{~cm}, \lambda=10.5 \mathrm{~cm}\right)$ energized at $40 \mathrm{MW}$ with 0.0032 duty cycle $(128 \mathrm{KW})$ wi17 have deposited in it $88 \mathrm{KW}$ total heat power, or about $29 \mathrm{KW} / \mathrm{m}$ The temperature rise of a disc aperture near the input of the guide ( $2 a=1.034$ in, $2 b=3.284$ in) by eq $(9)$ will be about $19.4^{\circ} \mathrm{C}$, where $R_{s}=.0139$ ohms/square and $K=4$ watts $/ \mathrm{cm} /{ }^{\circ} \mathrm{C}$. The heat load corresponds to about $500 \mathrm{~W}$ per disc. By the conditions proposed in deriving eq(11) the temperature rise of the disc aperture will be $29^{\circ} \mathrm{C}$ which is in reasonable agreement with the magnitude estimated from the complicated calculation.

The consequences of a large temperature gradient in the disc is that it may warp or distort, permanently de-tuning the waveguide in addition to recoverable thermal de-tuning. There are two stresses developed in a cylindrical plate by temperature gradients: 4

$$
\begin{aligned}
& S_{r}(r)=\alpha E\left[\frac{1}{b^{2}} \int_{a}^{b} r T(r) d r-\frac{1}{r^{2}} \int_{a}^{r} r T(r) d r\right]_{(12)} \\
& S_{\theta}(r)=\alpha E\left[\frac{1}{b^{2}} \int_{a}^{b} r T(r) d r+\frac{1}{r^{2}} \int_{a}^{r} r T(r) d r-T(r)\right]
\end{aligned}
$$

$S_{r}$ being the radial stress, $S_{\theta}$ the circumferential, $a$ the thermal coefficient of expansion, $E$ Young's modulus of elasticity, $b$ the outer radius of the disc, a the inner, and $T(r)$ the temperature distribution from eq $(7)$.

Quite evidently, the thermal problem can also be solved by means of a computer program 5 or, alternatively, on an analague resistor board. 6

The boundary temperature, $T_{0}$, is presumably established by means of a coolant flow rate sufficient to carry' away all heat generated in the steady state,

$$
P(K W)=0.265(G P M) \Delta T(\operatorname{deg} C)
$$

where $P(K W)$ is the average power removed, in kilowatts $\mathrm{GPM}$ is the flow rate, in gal/min

$\Delta T$ is the temperature rise of the coolant, in $\operatorname{deg} \mathrm{C}$

The system operating temperature should be higher than any ambient temperature; otherwise, the cooling system will be pumping heat from the accelerator tunnel and/or moisture may collect on the machine. About $115^{\circ} \mathrm{F}$ is conventional.

The story is not quite as simple as that outlined above. E. L. Chu has considered the consequences of the observation that the heat generated during an RF pulse cannot migratc more than a few 'skin' depths into the metal before the end of the pulse. This situation engenders a degenerate (or run-away) condition; owing to the increased skin temperature subsequent pulses will encounter a higher skin resistance and therefore the heat generated will be greater, raising the skin temperature still further, etc. Chu's results are too complicated to summarize briefly, for which reason the reader is referred to his account.?

The boundary condition stated in eq(1), while necessary to establish steady state for the system, oversimplifies the details of the waveguide temperature. Convective coolant flow is characterized by being either laminar (viscous) or turbulent, but in either case there is a stagnant film of fluid adhering to the metallic surface, across which there exists a temperature drop. Heat transfer is clearly more effective when the mixing action of turbulent flow causes the heat to be distributed throughout the coolant, but heat transfer is reduced owing to conduction through the film whose thickness is not easily defined. To circumvent this situation, the heat power transferred per unit area through the $f i 1 m$ of thickness 7 may be written 8

$$
\frac{P}{A}=\frac{K\left(T_{1}-T_{2}\right)}{1}=h\left(T_{1}-T_{2}\right)
$$

$h=K / 1$ being a film coefficient of heat transfer, usually determined from Nusselt's number. While the determination of these details are beyond the scope of this paper, being treated in any reasonable text on heat power engineering, the consequence is that the waveguide will be hotter than the coolant and not necessarily by a fixed amount (because the viscosity of the fluid depends upon its temperature). 
A slightly known relationship, used for practical engineering calculation is

$$
N_{u}=0.0225(\operatorname{Re})^{.8}(\mathrm{Pr})^{.4}
$$

Re being Reynold's number ( $\left.D v_{\rho} / \mu\right)$, Pr Prandtl's number $(\mu \mathrm{c} / \mathrm{K})$ and Nu Nusselts number $(h \mathrm{ho} / \mathrm{K})$, where $D$ is the pipe (waveguide) diameter, $k$ the thermal conductivity of the fluid, $p$ its density, $\mu$ its viscosity, $c$ its specific heat and $v$ its flow velocity (greater than critical). The critical velocity occurs when Reynold's number is about 2300 (dimensionless). Inconveniently, the calculated desion for clean, new pipe is quite in error when the conduits become fouled with scale, so that the designer ultimately is forced to rely on engineering judgment.

Normally, a waveguide is tuned under isothermal conditions and the coolant flow design cannot preserve that situation. The two most widely used coolant schemes are shown in figure 1. The 'cooling tube' plan permits counter current flow to be more easily realized the sheath plan permits greater flow rates. On the other hand, high flow rates, achieved by means of high velocities, may produce vibration. The physical mechanism of fluid flow vibration is not clearly established. In one type it is thought that there are coolant passage and manifold pressure oscillations at very low frequencies which perpetuates itself by alternately increasing and decreasing injection pressure thereby varying coolant flow which, in turn, varies coolant chamber pressure. Another type at low frequencies is thought to be caused by noise excitation of the natural frequencies of metal parts such as the waveguide, pipe lines and structural parts. It is possible that some vibration may be associated with acoustic resonances of the coolant passage. Vibration may result in system failure by metal "fatigue," as well as producing phase modulation in the accelerator. As a general rule flow rates should be less than $7 \mathrm{ft} / \mathrm{s}$ to avoid vibration. In addition, high pressure systems will de-tune the waveguide nonuniformly, the cylindrical cavity walls being permanently defarmed, owing to pressure 'creep.'

The consequences of heat losses in the structure have not been accepted supinely, without ingenious suggestions to thwart deleterious effects: 1) Principal, and most obvious, is the use of coolant passages in the disc; 2) It has also been proposed to perform the usual perturbation tuning of the waveguide after permitting it to distort itself at high average power; 3) It is also possible to increase the disc thickness, accepting reduced shunt impedance per unit length;4) A larger aperture would reduce the attenuation per unit length and thereby the heat deposition (the loss in shunt impedance ( $\mathrm{rL}$ ) can be compensated by increased length per section); 5) Where applicable, a lower operating frequency will entail larger scale structures and will have lower attenuation per unit length; 6) Some slight decrement in surface resistivity results from surface finish; 9 a 15 -micro-inch finish is a practical limit. Acid cleaning dip results in removal of machine tool marks and is equal to rouge or chalk polishing.

\section{REFERENCES}

1. E. L. Chu and W. Hansen, The Theory of Disq-Loaded Waveguides, JAP 18,996 (1947).

2. B. Friedmann, Frinciples and Techniques of Applied Mathematics, wiley, NY (1956) ch 3.

3. $\int x J_{1}^{2}(x) d x=\frac{x^{2}}{2}\left[J_{0}^{2}(x)+J_{1}^{2}(x)\right]-x J_{0}(x) J(x)$

$\int x \ln \frac{a}{x} J_{1}^{2}(k x) d x=\frac{x^{2}}{2}\left[j_{0}^{2}(k x)+J_{1}^{2}(k x)\right]\left(1+\ln \frac{a}{x}\right)$

$-\frac{x}{2 k}\left(\frac{1}{2}+\ln \frac{a}{x}\right) J_{0}(k x) J_{1}(k x)+\frac{1}{4 k^{2}} J_{0}^{2}(k x)$

4. Timoshenko, Theory of Elasticity (1934), p. 142.

5. S.Schriber, et al., Additions and Improvements to the RF Cavity Code SUPERFISH. IEEE Trans. Nuc. Sci., NS-30(4), 3545 (1983); J. Mckeown, Heat Transfer, Thermal Stress Analys is and the Dynamic Behavior of High Power RF Structures, IEEE Trans. Nuc. Sci., NS-30(4), 3593 (1983).

6. G. Liebmann, Precise Solution of Partial Differential Equations by Resistance Networks, Nature 164 , 149 (1949); J. R. Hechte1, Ein Widerstandsnetzwerk zur Loesung der Poissonschen Gleichung, Die Telefunken-Roehre, p. 38 (Feb. 1955); J. Hechtel and $\mathrm{J}$. Seeger, Accuracy and Limitations of a Resistor Network Used for Solving Laplace and Poisson Equations, Proc. IRE, 933 (1961).

7. Linear Electron Accelerator Studies, Status Report (1 Oct - 31 Dec, 1960) ML Report 785(1961) Hansen Lab. Stanford University.

8. I. Newton, Proc. Roy. Soc. (London) 22, 824 (1701).

9. R. C. Lending, ProC. NEC 11 (1957).

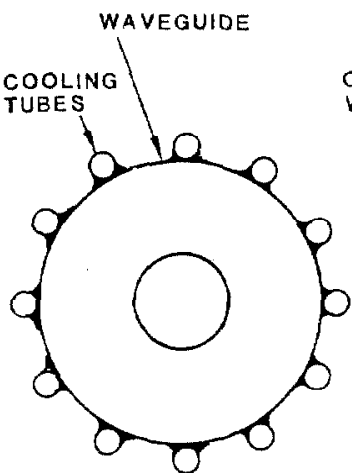

COOLING TUBE

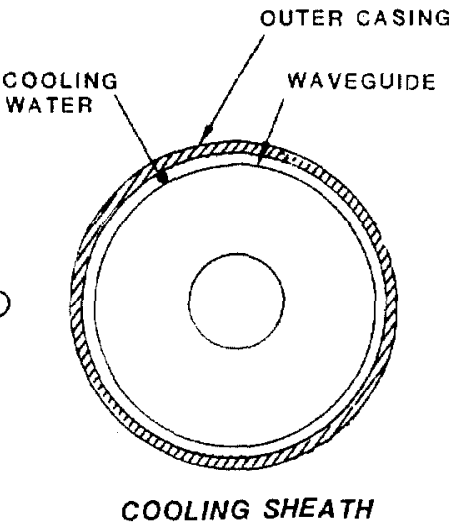

Figure 1 